\title{
Haptic subitizing across the fingers
}

\author{
Myrthe A. Plaisier • Jeroen B. J. Smeets
}

Published online: 9 April 2011

(C) The Author(s) 2011. This article is published with open access at Springerlink.com

\begin{abstract}
Numerosity judgments of small sets of items $(\leq 3)$ are generally fast and errorfree, while response times and error rates increase rapidly for larger numbers of items. We investigated an efficient process used for judging small numbers of items (known as subitizing) in active touch. We hypothesized that this efficient process for numerosity judgment might be related to stimulus properties that allow for efficient (parallel) search. Our results showed that subitizing was not possible forraised lines among flat surfaces, whereas this type of stimulus could be detected in parallel over the fingers. However, subitizing was possible when the number of fingers touching a surface had to be judged while the other fingers were lowered in mid-air. In the latter case, the lack of tactile input is essential, since subitizing was not enabled by differences in proprioceptive information from the fingers. Our results show that subitizing using haptic information from the fingers is possible only whensome fingers receive tactile information while other fingers do not.
\end{abstract}

\section{Keywords Numerosity judgement $\cdot$ Tactile $\cdot$ Fingers}

From vision, it is known that observers can judge the number of items in a set quickly and errorfree for small sets of items (less than three), while response times and error rates increase for larger numerosities (e.g., Atkinson, Campbell, \& Francis, 1976; Mandler \& Shebo, 1982; Trick \& Pylyshyn, 1994). It has been suggested that judgments of small numerositiesare accomplished through a fast process referred to as subitizing, while the slower process of counting is used for larger

M. A. Plaisier $(\square) \cdot$ J. B. J. Smeets

Faculty of Human Movement Sciences, VU University,

Van der Boechorststraat 9,

1081 BT Amsterdam, The Netherlands

e-mail: M.Plaisier@fbw.vu.nl numerosities (Kaufman, Lord, Reese, \& Volkmann, 1949). As a result, the function describing response time as a function of the number of items typically shows an upward bend at the transition between the subitizing and counting ranges. It is not yet clear what kind of process subitizing actually is. It has been suggested that subitizing is an accurate estimation process that can be used until the ratio between subsequent numerosities becomes smaller than the Weber fraction of $25 \%$ for visual number discrimination (Ross, 2003). However, recently it has been shown that thisexplanation does not hold (Revkin, Piazza, Izard, Cohen, \& Dehaene, 2008). Also, the idea that subitzing is preattentive and counting attentive has been contradicted by recent results showing that subitizing requires attention (e.g., Burr, Turi, \& Anobile, 2010; Olivers \& Watson, 2008; Railo, Koivisto, Revonsuo, \& Hannula, 2008). Whatever the underlying mechanism for judgments of small numerosities is, it is more efficient than counting and is not limited to the visual modality.

Although subitizing was first discovered in vision, more recently it has also been shown to exist in touch for up to three items. Subitizing was first shown for passive touch (touch without active exploration; Riggs et al., 2006). In that study, subjects had to determine the number of fingers stimulated by pins pressed onto the fingers. Subitizing has been shown to exist in active touch as well. In this case, subjects had to report the number of shapes grasped together in the hand (Plaisier, Bergmann Tiest, \& Kappers, 2009a, 2010a, 2010b). Although response time slopes for numerosity judgment in touch are generally larger than those in vision, processing of numerosity information is strikingly similar between the two modalities (Plaisier, Bergmann Tiest, \& Kappers, 2010c).

In some aspects, the distinction between counting and subitizing resembles the distinction between parallel and serial search that exists in both vision and haptics. Serial 
search indicates that all items in the set are processed subsequently inorder to find a certain target item, while parallel search indicates that items in the set can be processed simultaneously. As a consequence, parallel search yields fairly small slopes for response time as a function of set size. It has been shownthat subitizing can also occur in the presence of distractors, as long as the target-distractor combination yields parallel search (Trick \& Pylyshyn, 1993). This was, however, the case only for small numbers of distractors (less than four), and the subitizing slopes always increased when distractors were added.

For haptic search, it has been shown that an edge can be detected in parallel, both when several three-dimensional objects are grasped in the hand (Plaisier, Bergmann Tiest, \& Kappers, 2009b) and when the fingers are pressed onto a raised line (Lederman \& Klatzky, 1997; Overvliet, Smeets, $\&$ Brenner, 2007b). A combination of ellipsoids and spheres yields serial search, whereas a combination of spheres and cubes yields parallel search (Plaisier et al., 2009b). For haptic numerosity judgments, effects similar to those for vision have been found when subjects had to judge the number of spheres grasped in the hand, with either cubes or ellipsoids added as distractors (Plaisier, Van't Woud, \& Kappers, 2011). When spheres were judged in the presence of an ellipsoid, subitizing was not possible. In contrast, subitizing waspossible for judging the number of spheres in the presence of a cube. Adding a single distractor already led to an increase of the subitizing slope from $200 \mathrm{~ms} /$ item to $600 \mathrm{~ms} /$ item. So, adding distractors impairs or even prohibits subitizing in both vision and touch, but this depends on whether the target-distractor combination allows for parallel search.Given the similarities between the two types of search and the two types of numerosity judgments, we hypothesised that subitizing should be possible when the type of the item of which the numerosity has to be judged can be detected in parallel over the fingers.

In Experiment 1 we tested this hypothesis by asking subjects to press their fingers onto a set of flat surfaces and raised lines in order to determine the number of fingers touching a raised line. The results from Experiment 1 refuted our hypothesis. Therefore, we investigated the roles proprioceptive and tactile information in two further experiments.

\section{Experiment 1}

\section{Introduction}

It has been shown that the presence of a horizontal raised line among empty pieces of swell paper can be detected in parallel across the fingers (Overvliet et al., 2007b). In contrast, a vertical raised line among horizontal raised lines cannot be detected in parallel and yields serial search. Since subitizing is more efficient than the serial process of counting, we hypothesizedthat subitizing occurs when the targets can be detected in parallel across the fingers. In this experiment, wedetermined whether this is indeed the case by asking subjects the determine the number of horizontal raised lines among empty pieces of swell paper.

\section{Method}

Participants Eight employees (one male, all right-handed) of the Faculty of Human Movement Sciences of VU University participated in the experiment. All subjects were naive as to the purpose of the experiment and performed all three of the experiments in a counterbalanced order. The experiment was part of a program that has been approved by the ethics committee of the Faculty of Human Movement Sciences at VU University.

Setup and stimuli Items were placed underneath the fingers of both hands. The thumbs were not included in the experiment; therefore, the maximum number of items was eight. The items were placed on top of pressure-sensitive sensors that were triggered by placing a finger on top of an item. Response time measurement was started as soon as any of the sensors was triggered, and it was terminated with a vocal response. We had only six pressure sensors available; therefore, two items (and consequently fingers) did not have a sensor underneath. Because it could be that subjects systematically lowered some fingers more quickly than they did the other fingers, we varied the position of the sensors between subjects and also halfway through an experiment. Response times were measured with an accuracy of $24 \mathrm{~ms}$.

The items consisted of pieces of swell paper $\left(\mathrm{ZY}^{\circledR}{ }^{\circledR}\right.$ TECH2) with horizontally oriented raised lines (length of $2 \mathrm{~cm}$ and width of $2 \mathrm{~mm}$ ), as well as flat pieces of swell paper. The raised lines were placed under the finger pads of the subject such that the lines could be touched when the fingers were lowered (Fig. 1a). Subjects were instructed to report the number of raised lines presented. Subjects were explicitly instructed to move all their fingers downward simultaneously after the experimenter indicated that they could start a trial. Except for moving all the fingers downward simultaneously, there were no restrictions on exploratory movements. Subjects tended to make small finger movements over the items during a trial. A box with a small opening for the arms was placed over the setup to obscure it from the participants' vision.

Experimental design In all of the experiments, subjects were seated with their fingers elevated above the items. When the experimenter gave a signal, they placed each finger on an item and did so for all fingers simultaneously. They were instructed 
a

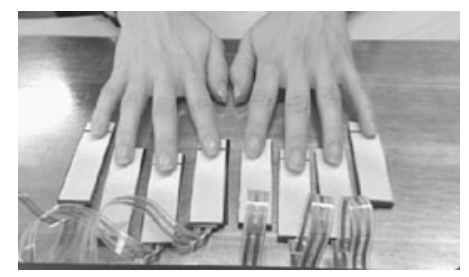

b

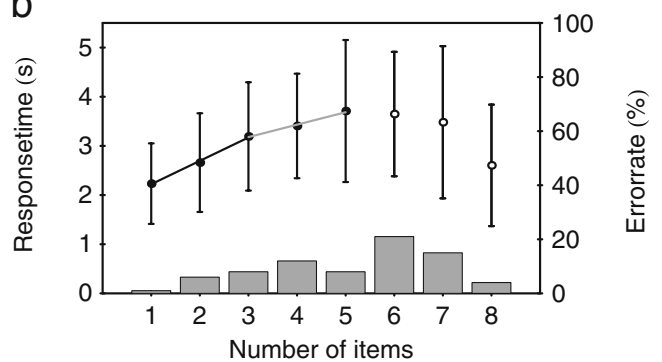

C

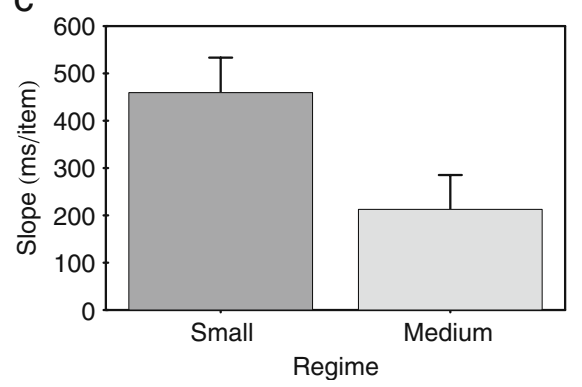

Fig. 1 Setup and results of Experiment 1. a Items consisted of raised lines and flat pieces of swell paper. b Response times averaged over subjects as a function of the number of items. Open symbols indicate large numerosities and were not included in the regression analysis. The errorbars indicate the standard deviation between subject means. The solid lines represent linear regression to the response times averaged over subjects for small (black line) and medium (gray line) numerosities. Error rates are indicated by the gray bars. c Slopes from the single-subject response times averaged over subjects for small and medium numerosities. Error bars indicate the between-subjects standard errors

to call out the correct number of items as quickly as possible. After each trial, the subjects received feedback on whether the answer was correct. To ensure the same number of correct trials for each numerosity, error trials were repeated at the end of the experiment. The presented numbers of items were one, two, three, four, five, six, seven, and eight. Each numerosity was presented 10 times. Before an experiment was started, subjects performed at least 20 practice trials, and practice continued until 10 trials in a row were correct. It was never necessary to exceed 25 practice trials.

Analysis Only response times from correctly answered trials were analyzed. To compare our results with those reported by Riggs et al. (2006), we adopted a similar analysis method. We divided our data into three numerosity regimes. Small number of items (one to three items), medium number of items (three to five), and large numbers of items (larger than five). We made this subdivision because subitizing is generally found for up to three items in haptics. Counting is always used for numerosities larger than three items. Because, in our experiment, the maximum number of items was restricted by the number of fingers, subjects could start to determine the number of nontarget items and subtract it from the maximum. This strategy could lead to a decrease in the response times for the largest numerosities. For numerosities of five or larger, it could become faster to use the described strategy than to judge the number of target items. This is the reason why, in the study of Riggs et al. (2006), in which 10 fingers were used, only response times for up to 6 fingers were analyzed. Because our data showed decreasing response times after five items, only numerosities up to five were included in our regression analysis. Linear regression was performed on the single-subject response times from one to three items and on those from three to five items. When there is subitizing for small numerosities, the slope for the response times from one to three items should be smaller than that for three to five items. One-tailed paired $t$-tests were performed on the slope values to test whether the slope for small numerosities was smaller than that for medium numerosities.

Because error trials were repeated at the end of the experiment and errors were made mainly for larger numerosities, we tested whether there was a learning effect. To this end, the response times were split into a first and a second half. There was no significant decrease between trials from the first and second halves in any of the experiments (paired samples $t$-test, $t(7) \leq 0.9, p \geq 0.4$ ).

\section{Results}

Response times averaged over subjects are shown as a function of the number of items in Fig. 1b. The bars indicate the error rates. It can be seen that the response times increase up to about five items and then decrease again. Linear regression was performed on the singlesubject response times for one to three items and three to five items (Fig. 1c). This yielded $450 \pm 70( \pm S E) \mathrm{ms} / \mathrm{item}$ for small numerosities and $210 \pm 70 \mathrm{~ms} /$ item for the medium numerosities. Contrary to our hypothesis, the slope for small numbers of items was thus not smaller than the slope in the medium numerosities range, $t(7)=0.1, p>.9$.

\section{Discussion}

Response times decreased after five items, indicating that, indeed, subjects counted the empty pieces of swell paper, instead of the raised lines, for large numerosities. This was also what subjects indicated upon debriefing and is in agreement with the findings of Riggs et al. (2006). 
Our results also show that numerosity judgmentswere not more efficient for one to three items than for three to five items. This indicates that subitizing was not possible for these stimuli. Apparently, subitizing is not always possible for tactile information presented to the fingers, even if the targets are distinguished by a highly salient tactile feature. This finding refutes our hypothesis that small sets of items differing in a feature that can be processed in parallel during search can also be subitized. In Experiment 2, we investigated whether subitizing is possible when the number of fingers touching an object has to be determined.

\section{Experiment 2}

\section{Introduction}

To investigate whether subitizing is possible when only the number of fingers touching an object has to be judged, wooden blocks were placed under some fingers, while no stimulus was placed under the other fingers. This means that some fingers did not receive any tactile stimulation at all.

\section{Method}

The same subjects as in Experiment 1 participated. The methodwas the same as that described in Experiment 1, except for the items, which in this case consisted of wooden blocks (height, $4 \mathrm{~cm}$ ). The hands rested on a $4.5-\mathrm{cm}$ raised surface such that the fingers were slightly elevated above the stimuli (Fig. 2a). Subjects moved their fingers down, touching the blocks with some fingers, while others were in midair. If the fingers accidentally touched a contact sensor on which no block was paced, the trial was discarded and repeated at the end of the experiment.

\section{Results}

It can be seen in Fig. $2 b$ that the response times show a sudden increase after three items and decrease again after five items. Again, linear regression was performed on the response times for one to three items and those for three to five items (Fig. 2c). This yielded $130 \pm 20 \mathrm{~ms} /$ item for small numerosities and $380 \pm 90 \mathrm{~ms} /$ item for medium numerosities. A paired samples $t$-test (one-tailed) showed that the slope in the subitizing range was smaller than the slope for the counting range, $t(7)=1.82, p=.02$.

\section{Discussion}

The fact that the slope for up to three items was smaller than that for three to five items is consistent with the idea that the process used to judge small numbers of items was more a
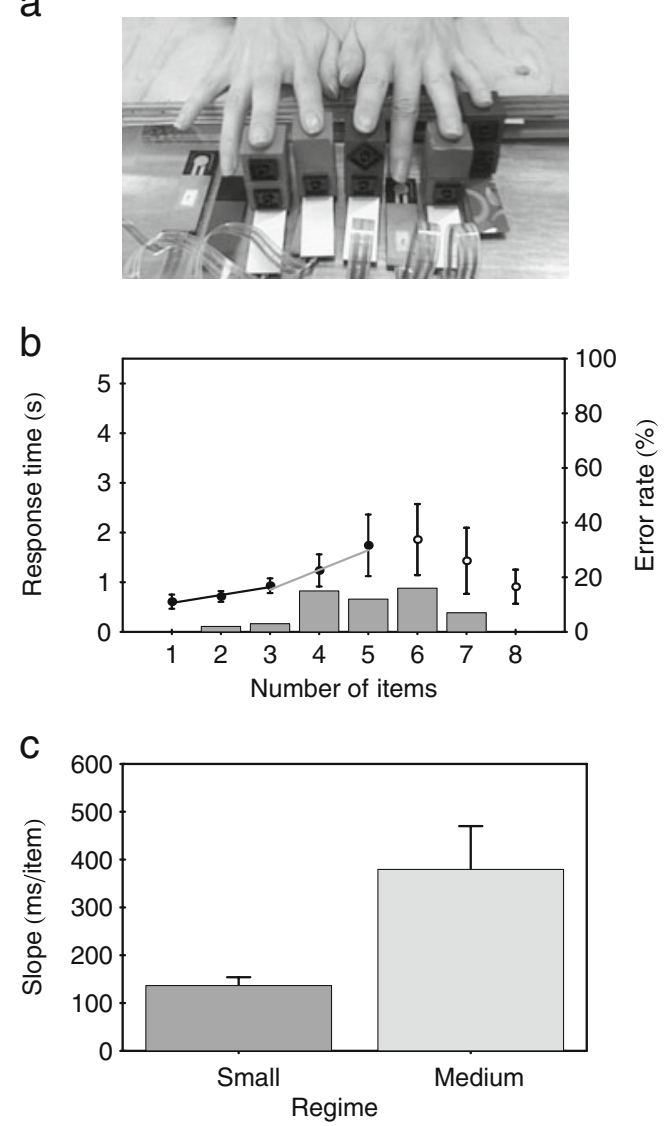

Fig. 2 Setup and results of Experiment 2. The meaning of the symbols is the same as in. a Items consisted of high wooden blocks. b Response times and error rates as a function of the number of items. $\mathbf{c}$ Slopes from the single-subject response times averaged over subjects for small and medium numerosities

efficient than the process used for larger numbers of items. This shows that the number of presented blocks could, indeed, be subitized. Our results suggest that subitizing is enabled when some fingers receive tactile information while others do not. However, it could also be that the difference in proprioceptive information from the fingers was used,since the fingers resting on the blocks were elevated, as compared with the fingers that were not resting on a block. To investigate whether this proprioceptive input was sufficient for subitizing, Experiment 3 was carried out.

\section{Experiment 3}

Introduction

In this experiment, high and low wooden blocks were placed under the fingers such that all the fingers were touching a block. This way, all the fingers received the same tactile information, but there were clear propriocep- 
tive differences between fingers touchinghigh and low blocks. The difference in block height was chosen such that the difference in proprioceptive information between the fingers was comparable to that in Experiment 2.

\section{Method}

The same subjects as in Experiment 1 and 2 participated. The methodwas again as described in Experiment 1, except that items consisted of high $(4 \mathrm{~cm})$ and low $(2 \mathrm{~cm})$ wooden blocks. The hands rested on a $4.5-\mathrm{cm}$ raised surface such that the fingers were slightly elevated above the blocks (Fig. 3a), and subjects moved their fingers down until all fingers were touching a block. Subjects were instructed to respond with the number of high blocks.

\section{Results}

It can be seen in Fig. $3 b$ that the error rates increase up to about six items and then decrease again. Linear regression

a

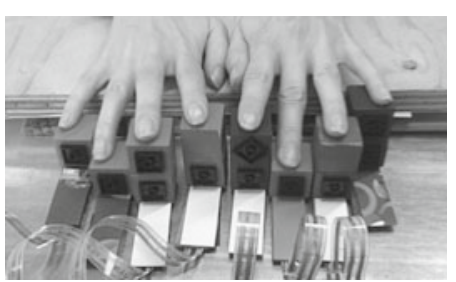

b
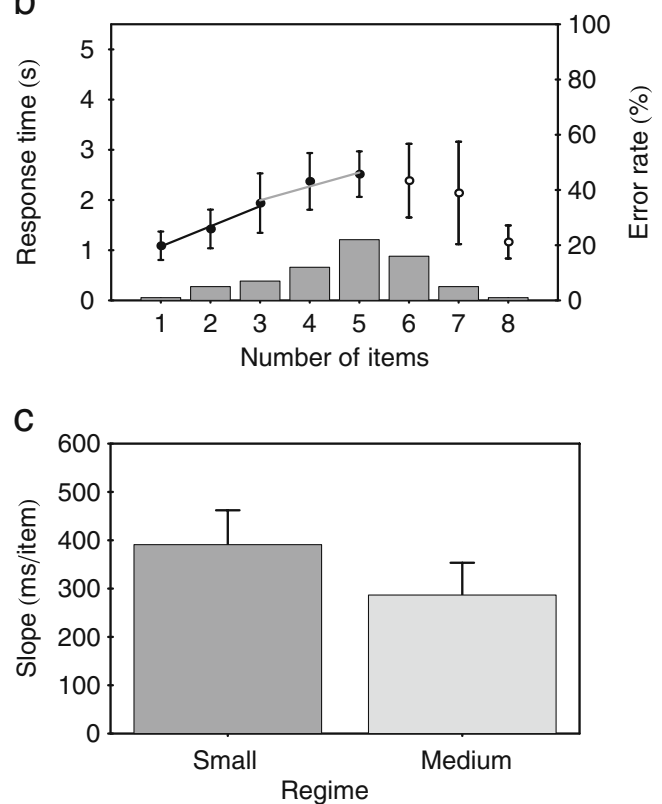

Fig. 3 Setup and results of Experiment 3. The meaning of the symbols is the same as in Figs. 2 and 3. a Items consisted of high and low wooden blocks. b Response times and error rates as a function of the number of items. c Slopes from the single-subject response times averaged over subjects for small and medium numerosities was performed on the response times for one to three items and those for three to five items (Fig. 3c). This yielded $390 \pm$ $70 \mathrm{~ms} /$ item for small numerosities and $290 \pm 70 \mathrm{~ms} /$ item for medium numerosities. As in Experiment 1, the slope for small numerosities was not smaller than the slope for medium numerosities, $t(7)=0.25, p=.8$.

\section{Discussion}

The fact that the slope for small numerosities was not smaller than the slope for medium numerosities indicates that there was no subitizing in this case. Therefore, we conclude that differences in proprioceptive information across fingers does not enable subitizing. This means that the absence of tactile stimulation on the fingers that were not being counted in Experiment 2 is crucial for subitizing to be enabled.

\section{General discussion}

Our results show that subitizing was possible only in Experiment 2, in which the number of fingers touching a wooden block had to be determined. In Experiment 1, where subjects had to determine the number of fingers touching a raised line,and in Experiment 3, in which subjects had to report the number of fingers touching a high wooden block, subitizing was not possible. Note that when subitizing was not possible, the subjects could count the number of items accurately at a rate of about $400 \mathrm{~ms} /$ item. Riggs et al. (2006) reported subitizing in the case where subjects had to judge the number of fingers stimulated with pins pressed onto the finger pads. In that study, the number of fingers receiving tactile information had to be determined. This iscomparable to the situation in Experiment 2 of the present study, in which some fingers did not receive any tactile stimulation.

These findings suggest that the number of objects can be subitized, but not a certain type of object among other objects, such as the raised lines among flat pieces of swell paper in Experiment 1 of the present study. Note that in Experiment 1, response times were remarkably large. In Experiment 1, there was essentially only tactile information, while in Experiment 2 and 3, there was also proprioceptive information. This does not mean that tactile information processing is generally slow. Braille reading, for instance, can be quite efficient. Note, however, that Braille reading involves pattern recognition of raised dots. Pattern recognition is much faster than numerosity judgment. In vision, it has been shown that subitizing-like response timescan be found up to six items, instead of four, as long as the dots are presented in fixed spatial patterns (Mandler \& Shebo, 1982). It is, however, possible that 
lateral scanning of tactile information, as in Braille reading, facilitates processing of the input. Indeed, tactile search is faster for lateral scanning with one finger than for static contact with multiple fingers (Overvliet, Smeets, \& Brenner, 2007a). Note that there is serial input of information in this case. Since subitizing is believed to be a parallel process, all items should be available at the same time.

The subitizing, as well as the counting, slopes found in Experiment 2 of the present study are somewhat smaller than those reported in previous studies for numerosity judgment. Riggs et al. (2006) reported a subitizing slope of $270 \mathrm{~ms} /$ item and a countingslope of $627 \mathrm{~ms} / \mathrm{item}$. In Experiment 2, we found a subitizing slope of $130 \mathrm{~ms} /$ item and a counting slope of $380 \mathrm{~ms} /$ item. In the study of Riggs et al.,pins were pressed onto the subjects' fingers, whereas in the present study, the subjects actively pressed their fingers onto the items themselves. It is possible that this active touch improved performance in the present study. When items consisted of objects actively grasped together in the hand, subitizing slopes ranged from $167 \mathrm{~ms} / \mathrm{item}$ to $200 \mathrm{~ms} /$ item, and counting slopes from $839 \mathrm{~ms} /$ item to 1,200 ms/item (Plaisier et al., 2009a, 2010a, 2010b). The subitizing slope of $130 \mathrm{~ms} /$ item found in the present study is thus only slightly smaller than subitizing slopes for objects grasped in the hand. The counting slopes were, in the latter case, however, considerably larger. This difference is probably due to the fact that counting of loose objects grasped in the hand is more demanding due to the difficulty of keeping track of which items have been counted already.

Visual subitizing slopes are, however, generally smaller (40-100 ms/item) than those found for touch (e.g., Akin \& Chase, 1978; Oyama, Kikuchi, \& Ichihara, 1981; Trick, 2008; Trick \& Pylyshyn, 1993). The reason for this difference in response time slopes between the modalities is not yet clear. It is, however, congruent with the fact that haptic search slopes are generally found to be larger than visual search slopes (Plaisier et al., 2009b). This indicates that this difference in response time slope values between the modalities is notspecific to numerosity judgment and originates from a more general difference in processing of information.

A possible explanation for the absence of subitizing in Experiment 1 and 3 is that cutaneous input from a flat surface can be regarded as a distractor. As was mentioned already in the introduction, adding distractors impairs or even prohibits subitizing in both vision and touch (Plaisier et al., 2011; Trick \& Pylyshyn, 1993). In vision, it has also been shown that some target-distractor combinations that yield parallel search cannot be subitized (Watson, Maylor, Allen, \& Bruce, 2007). The results of the present study can be interpreted in a similar way. A horizontal raised line among flat pieces of swell paper yields parallel search but cannot be subitized. Apparently, fingers touching a flat surface can be regarded as distractors (Experiments 1 and 3), while fingers that do not receive tactile input do not function as distractors (Experiment 2).

In vision, a set of concentric circles is an example of a stimulus that cannot be subitized but can be counted accurately. The same set of circles can, however, be subitized when spatially distributed. Trick and Pylyshyn (1993) suggested that items have to bespatially distributed to allow subitizing in vision. Trick and Pylyshyn (1993) suggested that items are individuated before the numerosity can be judged. Spatial distribution of the items probably allows for fast item individuation, which could be necessary to enable subitizing. In touch, we often judge the number of objects in our hand. In that case, there is no fixed spatial arrangement of the items, and they also do not need to be segmented from a background. An object is present or it is not; it is not a dot on a uniform surface, as is often the case in vision. Therefore, it would make sense that in touch,subitizing is used for judging the total number of objects being touched. Objects that are not present simply do not give tactile input. In this light, it makes sense that the number of fingers touching an object can be subitized but the mechanism breaks down when all fingers are touching a surface and receive some type of tactile input.

Author Note This research was supported by a VICI grant (MaGW 453-08-004) from the Netherlands Organisation for Scientific Research (NWO).

Open Access This article is distributed under the terms of the Creative Commons Attribution Noncommercial License which permits any noncommercial use, distribution, and reproduction in any medium, provided the original author(s) and source are credited.

\section{References}

Akin, O., \& Chase, W. (1978). Quantification of three-dimensional structures. Journal of Experimental Psychology. Human Perception and Performance, 4, 397-410.

Atkinson, J., Campbell, F. W., \& Francis, M. R. (1976). The magic number $4 \pm 0$ : A new look at visual numerosity judgments. Perception, 5, 327-334.

Burr, D. C., Turi, M., \& Anobile, G. (2010). Subitizing but not estimation of numerosity requires attentional resources. Journal of Vision, 10(6, Art. 20), 1-10.

Kaufman, E., Lord, M., Reese, T., \& Volkmann, J. (1949). The discrimination of visual number. The American Journal of Psychology, 62, 498-525.

Lederman, S. J., \& Klatzky, R. L. (1997). Relative availability of surface and object properties during early haptic processing. Journal of Experimental Psychology. Human Perception and Performance, 23, 1680-1707.

Mandler, G., \& Shebo, B. J. (1982). Subitizing: An analysis of its component processes. Journal of Experimental Psychology. General, 111, 1-22. 
Olivers, C. N. L., \& Watson, D. G. (2008). Subitizing requires attention. Visual Cognition, 16, 439-462.

Overvliet, K. E., Smeets, J. B. J., \& Brenner, E. (2007a). Haptic search with finger movements: Using more fingers does not necessarily reduce search times. Experimental Brain Research, 182, 427-434.

Overvliet, K. E., Smeets, J. B. J., \& Brenner, E. (2007b). Parallel and serial search in haptics. Perception \& Psychophysics, 69, 10591069.

Oyama, T., Kikuchi, T., \& Ichihara, S. (1981). Span of attention, backward masking, and reaction time. Perception \& Psychophysics, $29,106-112$.

Plaisier, M. A., Bergmann Tiest, W. M., \& Kappers, A. M. L. (2009a). One, two, three, many-Subitizing in active touch. Acta Psychologica, 131, 163-170.

Plaisier, M. A., Bergmann Tiest, W. M., \& Kappers, A. M. L. (2009b). Salient features in three-dimensional haptic shape perception. Attention, Perception, \& Psychophysics, 71, 421-430.

Plaisier, M. A., Bergmann Tiest, W. M., \& Kappers, A. M. L. (2010a). Grabbing subitizing with both hands: Bimanual number processing. Experimental Brain Research, 202, 507-512.

Plaisier, M. A., Bergmann Tiest, W. M., \& Kappers, A. M. L. (2010b). Haptic object individuation. IEEE Transactions on Haptics, 3, 257265.

Plaisier, M. A., Bergmann Tiest, W. M., \& Kappers, A. M. L. (2010c). Range dependent processing of visual numerosity: similarities across vision and haptics. Experimental Brain Research, 204, $525-537$.
Plaisier, M. A., Van’t Woud, M., \& Kappers, A. M. L. (2011). The effect of feature saliency on haptic subitizing. Experimental Brain Research, 209, 29-34.

Railo, H., Koivisto, M., Revonsuo, A., \& Hannula, M. M. (2008). The role of attention in subitizing. Cognition, 107, 82-104.

Revkin, S. K., Piazza, M., Izard, V., Cohen, L., \& Dehaene, S. (2008). Does subitizing reflect numerical estimation? Psychological Science, 19, 607-614.

Riggs, K. J., Ferrand, L., Lancelin, D., Fryziel, L., Dumur, G., \& Simpson, A. (2006). Subitizing in tactile perception. Psychological Science, 17, 271-272.

Ross, J. (2003). Visual discrimination of number without counting. Perception, 32, 867-870.

Trick, L. M. (2008). More than superstition: Differential effects of featural heterogeneity and change on subitizing and counting. Perception \& Psychophysics, 70, 743-760.

Trick, L. M., \& Pylyshyn, Z. W. (1993). What enumeration studies can show us about spatial attention: Evidence for limited capacity preattentive processing. Journal of Experimental Psychology. Human Perception and Performance, 19, 331-351.

Trick, L. M., \& Pylyshyn, Z. W. (1994). Why are small and large numbers enumerated differently? A limited-capacity preattentive stage in vision. Psychological Review, 101, 80-102.

Watson, D. G., Maylor, E. A., Allen, G. E. J., \& Bruce, L. A. M. (2007). Early visual tagging: Effects of target-distractor similarity and old age on search, subitization, and counting. Journal of Experimental Psychology. Human Perception and Performance, 33, 549-569. 